\title{
Utilizing mitochondrial events as biomarkers for imaging apoptosis
}

\author{
N Yivgi-Ohana ${ }^{1,2}$, M Eifer $^{1,2}$, Y Addadi $^{1,2}$, M Neeman $^{\star, 1}$ and A Gross ${ }^{1}$
}

Cells undergoing apoptosis show a plethora of time-dependent changes. The available tools for imaging apoptosis in live cells rely either on the detection of the activity of caspases, or on the visualization of exposure of phosphatidyl serine in the outer leaflet of the cell membrane. We report here a novel method for the detection of mitochondrial events during apoptosis, namely translocation of Bax to mitochondria and release of cytochrome $c$ (Cyt $c$ ) using bimolecular fluorescence complementation. Expression of split yellow fluorescent protein (YFP) fragments fused to Bax and Cyt $c$, resulted in robust induction of YFP fluorescence at the mitochondria of apoptotic cells with very low background. In vivo expression of split YFP protein fragments in liver hepatocytes and intra-vital imaging of subcutaneous tumor showed elevated YFP fluorescence upon apoptosis induction. Thus, YFP complementation could be applied for high-throughput screening and in vivo molecular imaging of mitochondrial events during apoptosis.

Cell Death and Disease (2011) 2, e166; doi:10.1038/cddis.2011.47; published online 2 June 2011

Subject Category: Cancer

Apoptosis, the process of programmed cell death, is fundamental to many biological events, including development, differentiation, regulation of the immune system and cancer. ${ }^{1}$ A precise balance of cell proliferation and cell death is required for tissue homeostasis, in which old or damaged cells are deleted and replaced by new cells. In cancer, apoptosis participates in the selection of cancer cells for aggressive phenotypes, including selection for resistance to hypoxia and anoikis-induced apoptosis. Many cytotoxic cancer therapies, such as DNA-damaging agents, radiotherapy and some of the new targeted drugs, are designed to induce apoptosis and cancer resistance, and escape mechanisms are associated with defects in the apoptotic machinery.

Apoptotic cells can be recognized by defined morphological changes: the cell shrinks, its chromatin condenses, the plasma membrane shows blebbing, and finally the cell is fragmented into compact membrane-enclosed structures. These morphological changes are a consequence of characteristic molecular and biochemical events occurring in an apoptotic cell, including activation of cysteine-dependent, aspartate-specific proteases (caspases) that coordinate and execute the process.

Several approaches to monitor apoptosis non-invasively have been developed, with varying degrees of sensitivity and specificity. Some rely on the structural changes occurring in an apoptotic tissue, which can be detected by ultrasound ${ }^{2}$ or diffusion-weighted $\mathrm{MRI}^{3}$ Other methods include reagents developed for monitoring activation of caspase proteases by fluorescence, ${ }^{4}$ bioluminescence ${ }^{5}$ or radiolabeled probes. ${ }^{6}$
Thus, caspase activity can be detected through enhanced photon emission after enzymatic cleavage of the sequence Asp-Glu-Val-Asp (or DEVD) by caspase-3 or caspase-7. ${ }^{7}$ Additional reagents use the exposure of phosphatidyl serine in the outer leaflet of the cell membrane of apoptotic cells, for retention and accumulation of targeted contrast media such as labeled Annexin- $\mathrm{V}^{8}$ or the $\mathrm{C} 2$ domain of synaptotagmin-I. ${ }^{9}$ These events can occur also in other, non-apoptotic, cellular processes. ${ }^{10,11}$

Members of the $\mathrm{Bcl}-2$ family are major regulators of the apoptosis process. ${ }^{12}$ Bax, a proapoptotic member of the family, resides in the cytosol in an inactive state, in which its $\mathrm{N}$ - and C-terminal parts are folded within the protein core. ${ }^{13,14}$ In response to an apoptotic signal, Bax translocates to the mitochondria and inserts into the outer mitochondrial membrane $\left(\mathrm{OMM} ;{ }^{15,16}\right)$. The translocation and membrane insertion of Bax are accompanied by conformational changes, which include exposure of its $\mathrm{N}$ - and $\mathrm{C}$-terminal parts. Insertion into the OMM triggers the homodimerization of Bax, resulting in mitochondrial outer membrane permeabilization (MOMP) and release of inter-membrane-space proteins such as cytochrome $c\left(\right.$ Cyt $\left.c ;^{17-19}\right)$. Once released, Cyt $c$ interacts with Apaf-1 to form the apoptosome, leading to caspase activation. ${ }^{20}$ Previously, fluorescence resonance energy transfer (FRET) was used to show that Bax interacts with the antiapoptotic $\mathrm{Bcl}-2$ protein at mitochondria; however, this technique was not used to monitor apoptosis. ${ }^{21}$

In the work presented here, we took advantage of the mitochondrial events to monitor apoptosis in cells and in living

\footnotetext{
${ }^{1}$ Department of Biological Regulation, Weizmann Institute of Science, Rehovot, Israel

${ }^{*}$ Corresponding author: M Neeman, Department of Biological Regulation, Weizmann Institute of Science, Rehovot 76100, Israel. Tel: +97 289342487 ;

Fax: + 9728934 6264; E-mail: michal.neeman @weizmann.ac.il

${ }^{2}$ These authors contributed equally to this work.

Keywords: programmed cell death; protein interaction; intravital microscopy; molecular imaging; mitochondria

Abbreviations: DEVD, Asp-Glu-Val-Asp; CP, cisplatin; caspases, cysteine-dependent, aspartate-specific proteases; Cyt c, cytochrome c; FRET, fluorescence resonance energy transfer; MOMP, mitochondrial outer membrane permeabilization; $\mathrm{mPT}$, mitochondrial permeability transition; OMM, outer mitochondrial membrane; ROS, reactive oxygen species; YFP, yellow fluorescent protein

Received 28.2.11; revised 18.4.11; accepted 19.4.11; Edited by P Salomoni
} 
animals. The experimental strategy was based on the proteinfragment complementation of yellow fluorescent protein (YFP). ${ }^{22,23}$ In this system, the $\mathrm{N}$ - and C-terminal fragments of YFP, which are genetically fused to candidate interacting proteins, will produce a fluorescent signal only if the fused proteins will reside in the same cellular compartment and bring the YFP fragments into close proximity. ${ }^{23}$ Based on molecular events during Bax activation, we developed a novel tool to image apoptotic cells, with very low background signal and significant amplification of fluorescence induced by apoptotic signals.

\section{Results}

Split YFP as a tool to image Bax activation/homodimerization in tumor cells. The background fluorescence signal provided by the split YFP system was evaluated by cloning the split YFP N-terminal fragment upstream to an $\mathrm{HA}$ tag (N-YFP-HA) and the C-terminal fragment (C-YFP) independently in the backbone of the EGFP expression vector. Human breast carcinoma (MCF-7) cells were transiently transfected with the two constructs and stained with anti-HA antibodies by immunofluorescence (IF; Figure 1a, top panel). A high YFP signal observed in HA-positive cells suggested that spontaneous complementation of the two fragments when expressed in the same cellular compartment was sufficient to induce fluorescence. However, when the YFP fragments were separated to different cellular compartments by fusing the N-YFP to the mitochondrial leader sequence cytochrome oxidase subunit-VIII (mito-N-YFP-HA), the levels of the YFP signal were significantly lowered (Figures 1a and b). When N-YFP and C-YFP were independently cloned upstream to HA-Bax, negligible YFP fluorescence was detected by microscopy and by the plate reader (Figures 1a and b). This low signal could be explained by the closed conformation of a

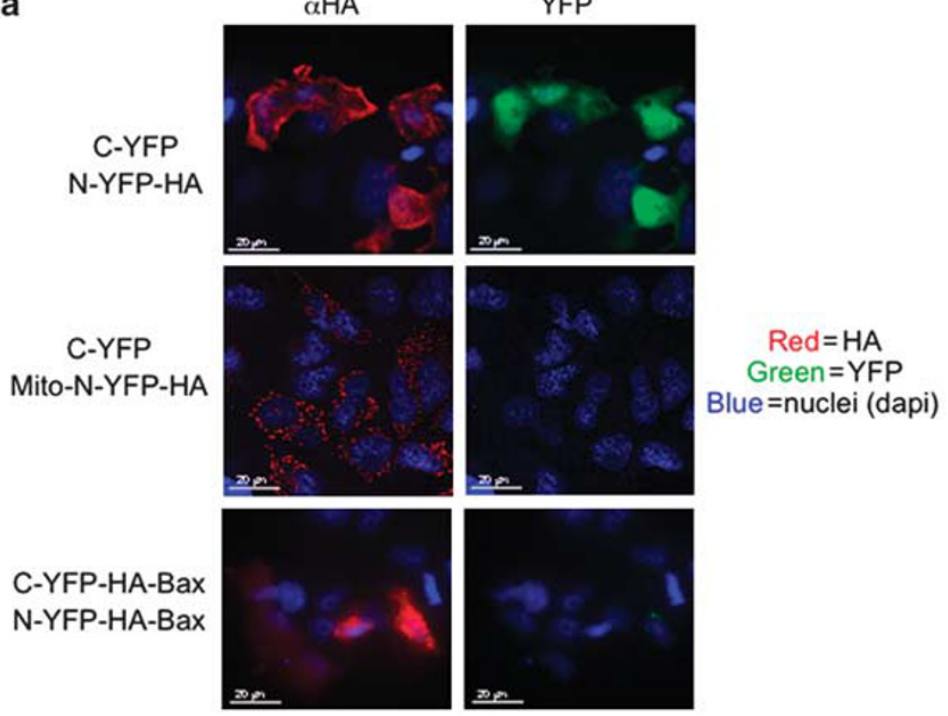

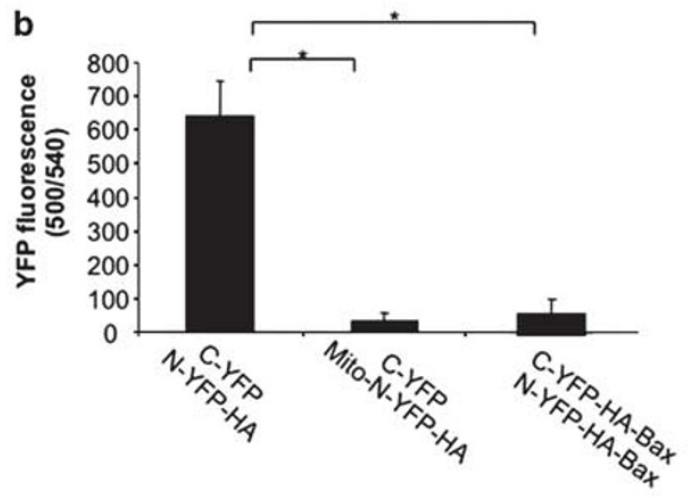

C

C-YFP-HA-Bax, N-YFP-HA-Bax
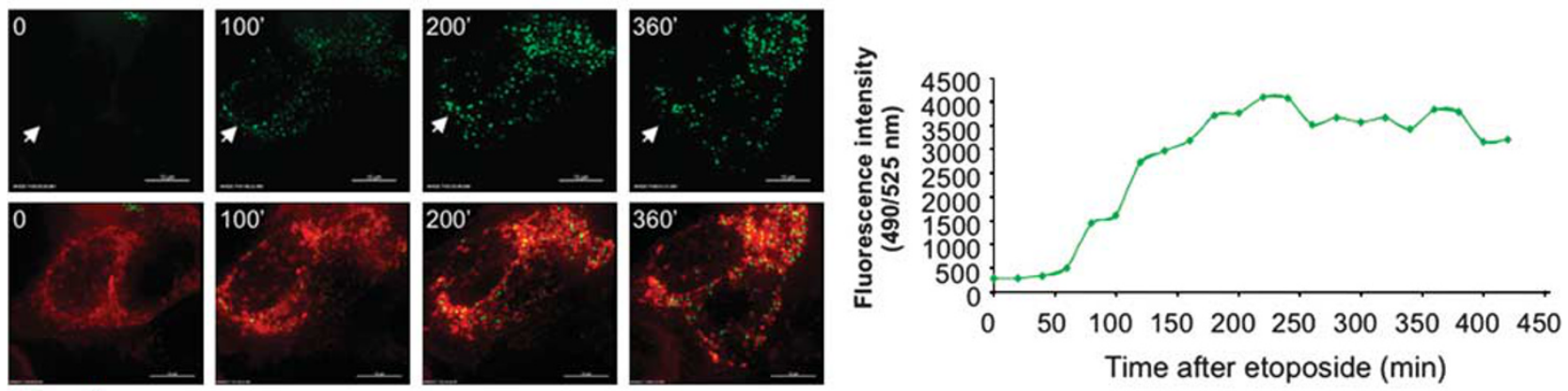

Figure 1 N-YFP-HA-Bax and C-YFP-HA-Bax generate a YFP signal in cells treated with etoposide. (a) MCF-7 cells were transiently transfected with either C-YFP and $\mathrm{N}-$ YFP-HA (top panel), C-YFP and Mito-N-YFP-HA (middle panel), or C-YFP-HA-Bax and N-YFP-HA-Bax (bottom panel). IF staining using anti-HA antibody was used to correlate the subcellular localization of the YFP fragments (red) with the YFP signal (green). (b) The bar graph depicts the difference in the YFP signal observed in panel a, measured using a fluorescent plate reader; $n=3,{ }^{*} P<0.005$. (c) MCF-7 cells were transiently transfected with N-YFP-HA-Bax, C-YFP-HA-Bax and mitochondrial mCherry. After $24 \mathrm{~h}$, the cells were treated with $100 \mu \mathrm{M}$ etoposide and followed using a DeltaVision microscope. $100 \mathrm{~min}$ after the addition of the death signal, a strong YFP fluorescence was detected (left panel). Right panel: YFP fluorescence intensity was measured for the cells in panel c using the SoftWoRx software (Applied Precision, Issaquah, WA, USA) 
cytosolic Bax in non-apoptotic cells, ${ }^{13,14}$ thus preventing YFP complementation.

If the assumption presented above is correct then an apoptotic signal resulting in the activation of Bax and its homodimerization should lead to an increase in the YFP signal at the mitochondria. To address this point, N-YFP-HABax and C-YFP-HA-Bax were co-expressed in MCF-7 cells and $24 \mathrm{~h}$ later the cells were followed by live-cell imaging. Indeed, YFP background levels in viable cells were very low (Figure 1c, left top image, time 0). Treating the cells with etoposide resulted in a significant increase in YFP fluorescence at the mitochondria (Figure 1c, top images; Supplementary Movie 1). The mitochondria in these cells were labeled with mito-mCherry (Figure 1c, bottom images). Dynamic analysis of the temporal change in YFP fluorescence intensity showed an $\sim 15$-fold increase in fluorescence following etoposide treatment (Figure 1c, right graph).

Bax is known to interact with the BH3-only BID protein, and thus we also analyzed the Bax-Bid split YFP pair. Expression of the Bax-Bid pair resulted in high background levels, and an apoptotic stimulus induced the translocation of the signal from the cytosol to the mitochondria, resulting in a maximal 2.1-fold increase in fluorescence (Supplementary Movie 2).

The split YFP system enables to monitor Bax conformational changes. The ability of the split YFP system to monitor the conformational activation of Bax was evaluated using a number of Bax mutants (Figure 2). The P13A mutant, which was reported to show elevated apoptotic activity, ${ }^{24}$ was detected in both the cytoplasm and the mitochondria of MCF-7 cells (left panels), and accordingly, YFP fluorescence was significantly higher than for wild-type Bax (right panels). The P168A mutant, which was reported to reside in the cytosol with an exposed $\mathrm{N}$-terminus, ${ }^{25}$ showed a high cytosolic YFP signal similar to the maximal YFP signal obtained with full-length YFP fused to the mitochondrial Smac protein. The S184V mutant, which was reported to reside at the mitochondria in healthy cells with a buried $\mathrm{N}$-terminus, ${ }^{26}$ showed a low mitochondrial YFP signal. Finally, it was reported previously that fusing a protein tag to the $\mathrm{C}$-terminus of Bax results in high levels of cell death. ${ }^{27}$ Indeed, cloning the split YFP in the C-terminus of Bax and its expression in MCF-7 cells resulted in high levels of cell death and maximal YFP fluorescence (Figure 2, right panel). Thus, the split YFP system enables to monitor the conformational changes associated with Bax activation.

Split YFP as a tool to image mitochondrial apoptosis. In search for a split YFP protein pair that will 'turn-on' once apoptosis has occurred at the mitochondria, we screened different protein pairs that can potentially target the YFP fragments to different cellular compartments in non-apoptotic cells and to the same compartment in apoptotic cells. Interestingly, protein pairs that are known to interact during apoptosis, such as the Cyt $c-A p a f-1$ and Smac-XIAP pairs, failed to show an increase in YFP fluorescence upon an apoptotic stimulus (Table 1). On the other hand, the Bax-Cyt $c$ and Bax-Smac pairs, which are not known to interact, showed a low background signal and a significant increase in YFP fluorescence upon apoptosis stimulation (Table 1, and see below).

In response to an apoptotic stimulus, Bax translocates to the mitochondria, where it induces the release of Cyt $c$. In an attempt to visualize Bax translocation using the split YFP system, we generated C-YFP-Flag-Bax and Cyt c-N-YFP$\mathrm{HA}$. IF analysis confirmed that in healthy cells Bax was cytosolic and Cyt $c$ resided in mitochondria, and that treatment with the TNF $\alpha$ death ligand resulted in the translocation of Bax to the mitochondria and release of Cyt $c$ into the cytosol (Figure 3a). Co-transfection of MCF-7 cells with C-YFP-HABax and Cyt $c-N-Y F P$ resulted in low background fluorescence (Figure $3 b$, top left panel, time 0 ). Treating the cells with $\mathrm{TNF} \alpha$ resulted in a significant increase in the YFP fluorescence intensity at the mitochondria (Figure 3b, top left panels; Supplementary Movie 3).

To correlate the increase in YFP fluorescence with apoptosis, we co-expressed in the same cells a modified mCherry caspase sensor, which includes the effector caspase consensus cleavage site (DEVD) and the transmembrane domain of $\mathrm{Bcl}-\mathrm{xL}$ (TD), which targets the fusion protein to the OMM. ${ }^{28,29} \mathrm{TNF}_{\alpha}$ treatment resulted in the release of

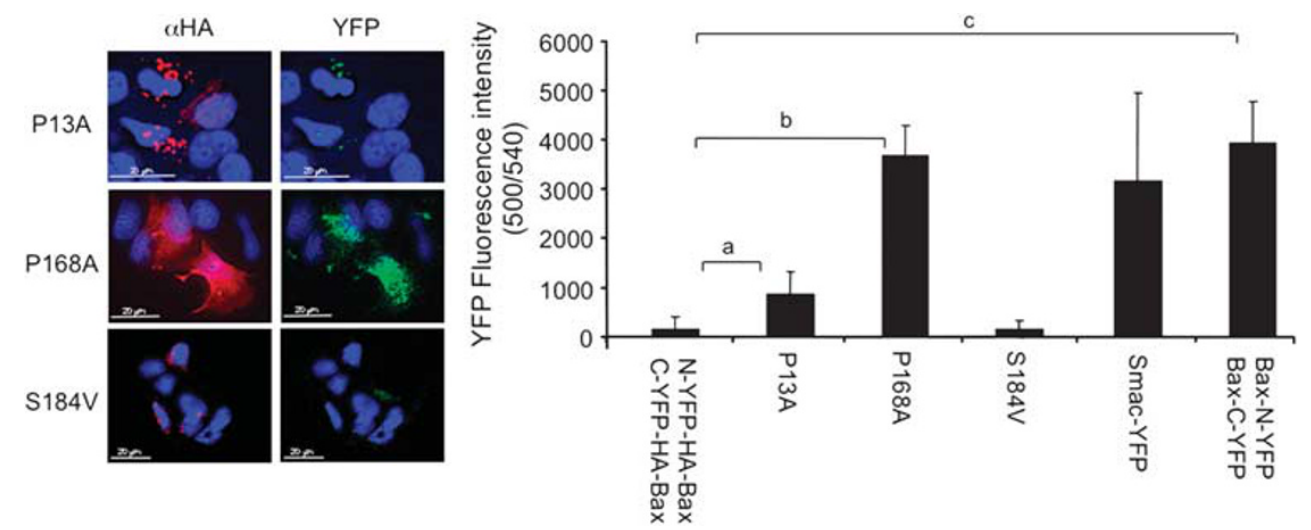

Figure 2 Split YFP as a tool to image Bax conformational change. C-YFP-HA and N-YFP-HA were independently cloned downstream or upstream to wild-type Bax or different Bax mutants. MCF-7 cells were transiently transfected and $24 \mathrm{~h}$ later the levels of YFP were measured either using a fluorescence plate reader (right panel) or by fluorescence microscopy, and correlated with anti-HA staining (red, left panel, $n=4 ;{ }^{a} P<0.05 ;{ }^{b} P<0.00001 ;{ }^{c} P<0.0005$ ) 
Table 1 Pairs of candidate interacting proteins were evaluated using the split YFP system for their ability to generate specific fluorescence upon induction of apoptosis in MCF7 cells

\begin{tabular}{|c|c|c|c|}
\hline \multicolumn{2}{|c|}{ Protein pairs } & \multicolumn{2}{|c|}{ YFP fluorescence } \\
\hline C-YFP & N-YFP & Baseline & Apoptosis \\
\hline $\begin{array}{l}\text { WD(APAF-1)-C-YFP } \\
\text { XIAP-C-YFP } \\
\text { C-YFP-Bax } \\
\text { C-YFP-Bax } \\
\text { C-YFP-Bax } \\
\text { C-YFP-Bax } \\
\text { Bid-C-YFP }\end{array}$ & $\begin{array}{l}\text { Cyt } c-N-Y F P \\
\text { Smac-N-YFP } \\
\text { MitoPLD-N-YFP } \\
\text { Cyt } c-N-Y F P \\
\text { Smac-N-YFP } \\
\text { N-YFP-Bax } \\
\text { N-YFP-Bax }\end{array}$ & $\begin{array}{l}\text { Low background } \\
\text { Low background } \\
\text { Low background } \\
\text { Low background } \\
\text { Low background } \\
\text { Low background } \\
\text { High background in cytosol }\end{array}$ & $\begin{array}{l}\text { No change } \\
\text { No change } \\
\text { No change } \\
\text { High increase in mitochondria } \\
\text { High increase in mitochondria } \\
\text { High increase in mitochondria } \\
\text { Translocation to mitochondria }\end{array}$ \\
\hline
\end{tabular}

Apoptosis was induced by TNF $\alpha$ or etoposide. Fluorescence was followed by live-cell imaging using a DeltaVision system.

a

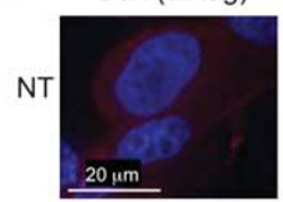

Cyt c $(\alpha \mathrm{HA})$

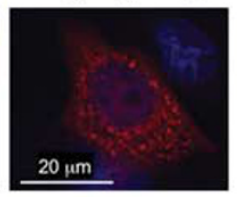

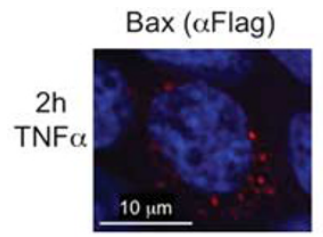

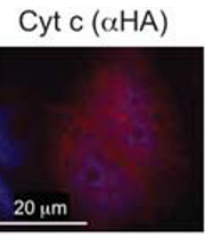

b C-YFP-HA-Bax, Cyt c-N-YFP
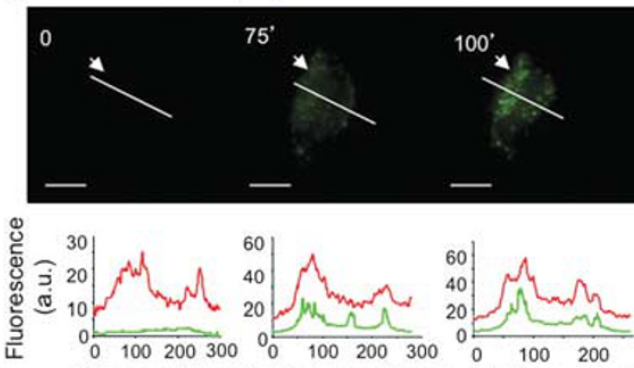

$125^{\prime}$
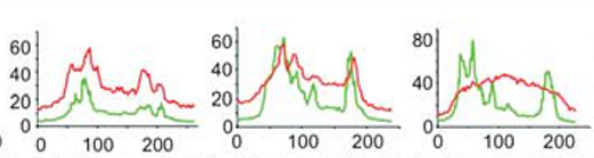

$150^{\prime}$

Distance (Pixels) Distance (Pixes) Distan
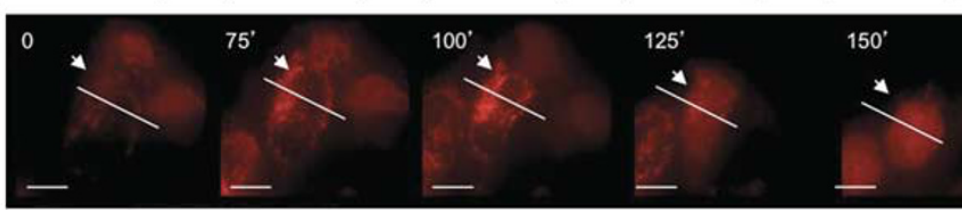

225

c mCherry-DEVD-Bcl-xl-TD

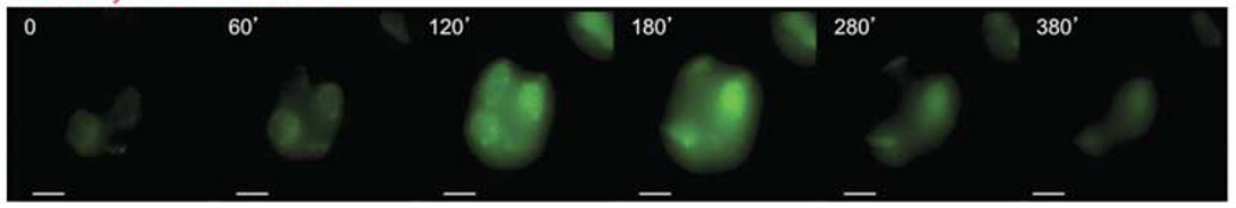

Figure 3 C-YFP-HA-Bax and Cyt $c-N-Y F P$ generate a specific YFP signal that correlates with caspase activation in cells treated with TNF $\alpha$. (a) MCF-7 cells were transiently transfected with C-YFP-Flag-BAX and Cyt c-N-YFP-HA. IF staining using anti-Flag and anti-HA antibodies, with or without TNF $\alpha$ (10 ng/ml) and actinomycin-D $(2 \mu \mathrm{g} / \mathrm{ml})$ treatment, shows the sub-cellular distribution of the proteins. (b) MCF-7 cells expressing C-YFP-HA-Bax, Cyt $c-N-Y F P$ and an mCherry caspase sensor were treated with TNF $\alpha$ and ActD, and imaged using a DeltaVision time-lapse microscope. The YFP signal increases at the mitochondria 75 min after the addition of TNF $\alpha$ (top left panel; bar, $10 \mu \mathrm{m}$ ). Fluorescence of mCherry is shown in the bottom panel (bar, $10 \mu \mathrm{m})$. YFP and mCherry fluorescence intensities along the marked line were measured using the ImageJ software (middle panel). Maximal YFP fluorescence relative to maximal mCherry fluorescence divided by the mean is plotted on the graph (top right panel). The induction of YFP signal was followed by the translocation of mCherry to the cytoplasm and correlated with caspase activity (low right panel). (c) MCF-7 cells expressing C-YFP-HA-Bax and Cyt $c-N-Y F P$ were treated with $30 \mu \mathrm{M}$ menadione and imaged using a DeltaVision time-lapse microscope (bar, $10 \mu \mathrm{m}$ )

mCherry into the cytoplasm shortly after the increase in mitochondrial YFP fluorescence (Figure 3b, compare bottom images to top images). The time scale of mCherry release into the cytoplasm relative to the increase in YFP signal is plotted in the left panel (graphs between the images) and right panel (top graph), respectively. The release of mCherry into the cytoplasm correlated with an increase in caspase activity (Figure 3b, bottom right graph). 
Another inducer of cell death through the mitochondria is menadione, which generates reactive oxygen species (ROS) through redox cycling. MCF7 cells transiently transfected with C-YFP-HA-Bax and Cyt $c-N-Y F P$ were treated with $30 \mu \mathrm{M}$ menadione and followed by live-cell imaging (DeltaVision; Figure 3c and Supplementary Movie 4). A significant increase in YFP was observed $120 \mathrm{~min}$ after treatment and peaked at $180 \mathrm{~min}$.

Detection of Fas-induced apoptosis of liver hepatocytes using the Bax-Bax and Bax-Cyt $\boldsymbol{c}$ split YFP pairs. Next we evaluated whether the expression of the Bax-Bax and the Bax-Cyt $c$ split YFP pairs by hepatocellular somatic gene transfer (using hydrodynamic DNA injection ${ }^{30}$ ) can be used to monitor apoptosis induced by anti-Fas antibody in liver hepatocytes. ${ }^{31}$ Male, 8- to 9-week-old FVB mice were hydrodynamically injected with either C-YFP-HA-Bax and Cyt $c-N-Y F P$ (Figure 4a), or N-YFP-HA-Bax and C-YFP$\mathrm{HA}-\mathrm{Bax}$ (Figure $4 \mathrm{~b}$ ), and $24 \mathrm{~h}$ later the mice were either left untreated or injected with a single i.p. dose of $0.55 \mu \mathrm{g} / \mathrm{g}$ anti-Fas antibody. Four hours after the anti-Fas antibody injection, two-photon microscopy showed the emergence of YFP-positive hepatocytes in the livers from the hydrodynamically injected mice, and the number of YFP-positive cells was elevated by the anti-Fas antibody injection (Figures $4 a$ and $b$, left and right panels). Histological staining of floating sections from the livers of Fas-injected mice with an anti-cleaved/active caspase- 3 antibody confirmed that a majority of the caspase-3-positive cells showed a strong YFP signal (Figure 4c, arrows). Similar results were obtained with the Bax-Cyt $c$ split YFP pair (not shown).

Stable expression of C-YFP-Bax and Cyt $\boldsymbol{c}$-N-YFP does not increase sensitivity to death signals. MCF-7 cells were infected with lentiviruses containing N-YFP-Bax and Cyt $c-N-Y F P$, stable clones were selected using blasticidine and the expression of the proteins was analyzed by Western blotting (Figure 5a). The levels of YFP upon etoposide treatment were determined by live-cell imaging (DeltaVision; Figure $5 b$ ). To assess whether overexpression of C-YFPBax and Cyt $c-N-Y F P$ results in hypersensitivity to apoptotic signals, we treated the cells with staurosporine, etoposide or a
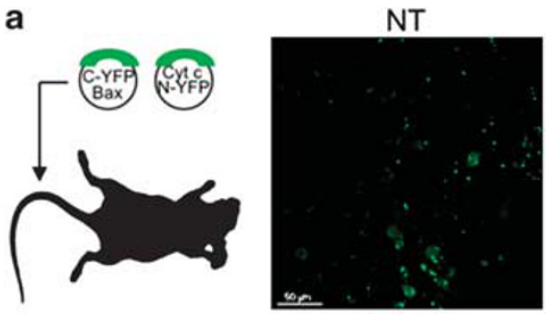

Bax-Cyt c pair

b

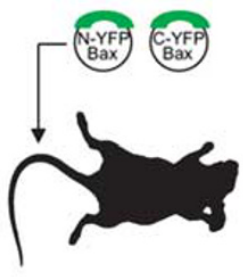

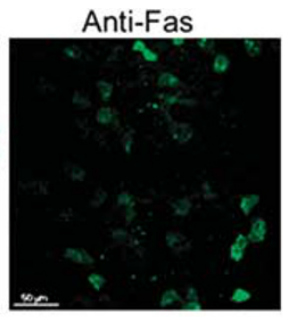

Anti-Fas

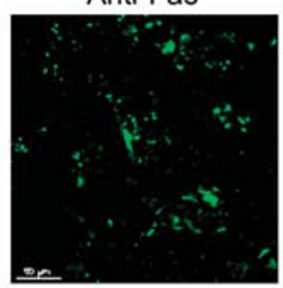

Bax-Bax pair

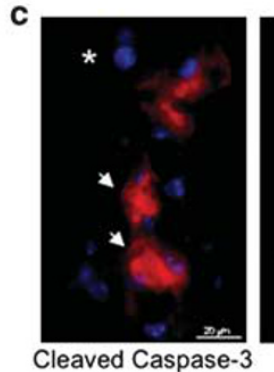

Cleaved Caspase-3

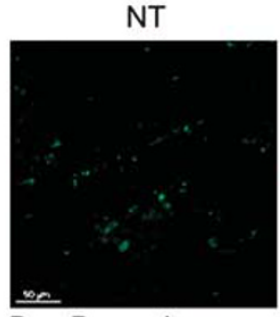

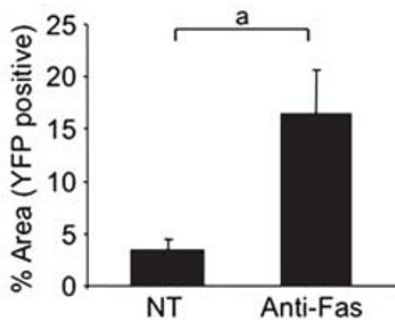
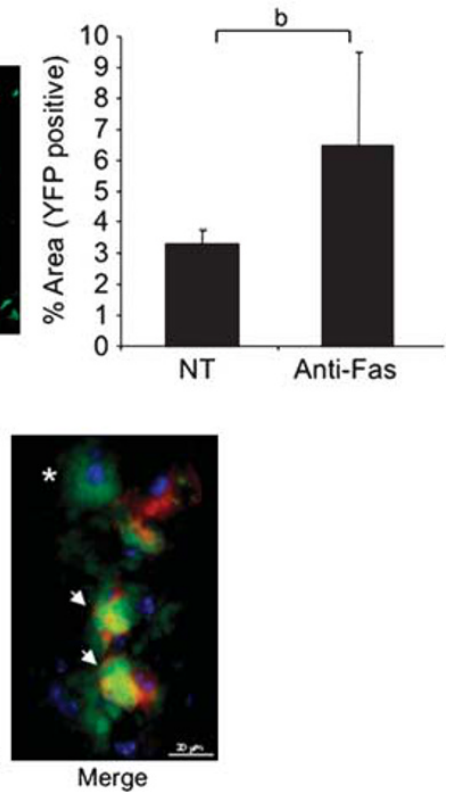

Figure 4 In vivo complementation of Bax-Bax and Bax-Cyt $c$ YFP in liver. FVB mice were i.v. injected with $30 \mu \mathrm{g}$ of a mixture of N-YFP-HA-Bax and C-YFP-HA-Bax (a) or C-YFP-HA-Bax and Cyt c-N-YFP (b). Twenty-four hours later the mice were left untreated $(n=2)$ or injected with a single i.p. dose of $0.55 \mu \mathrm{g} / \mathrm{g}$ anti-Fas antibody $(n=4)$. Four hours after anti-Fas antibody injection mice were killed and livers were immediately imaged, ex vivo, using a two-photon microscope, using the YFP channel $(850 \mathrm{~nm})$. The percentage of YFP-positive area was measured using the ImageJ software (right panel); ${ }^{\mathrm{a}} P<0.005 ;{ }^{\mathrm{b}} P<0.05$. (c) Histological floating sections of liver prepared from mice hydrodynamicaly injected with N-YFP-HA-Bax and C-YFP-HA-Bax were stained using an anti-cleaved/active caspase-3 antibody (red) and correlated with the YFP signal (green, arrows). *Indicates YFP-positive cells with no red staining, indicating Bax activation before caspase activation 
a

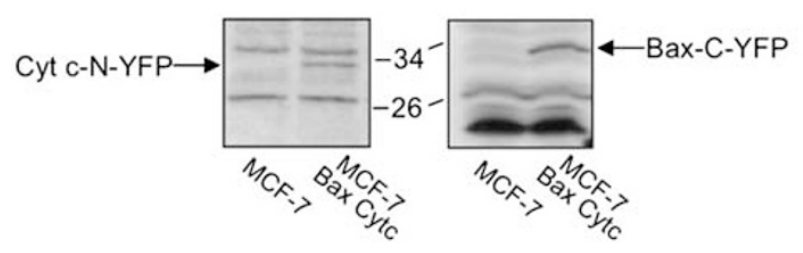

b

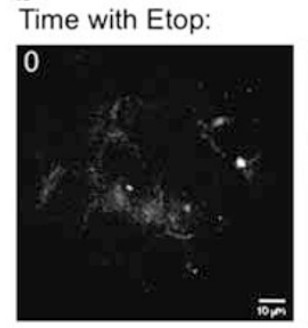

Cell death following Etop treatment
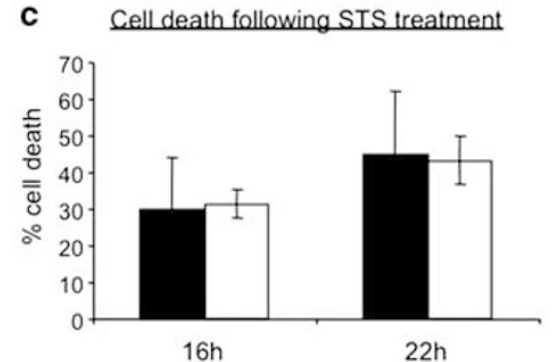

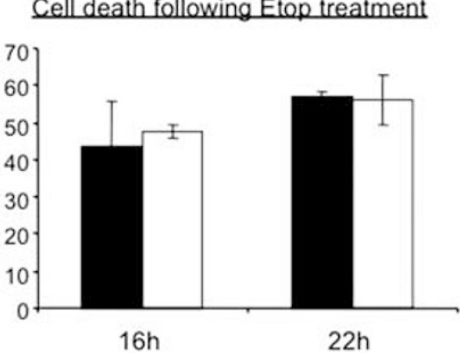

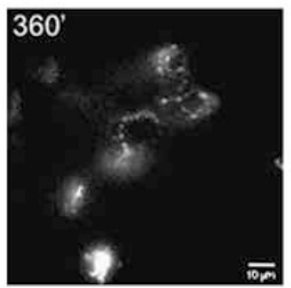

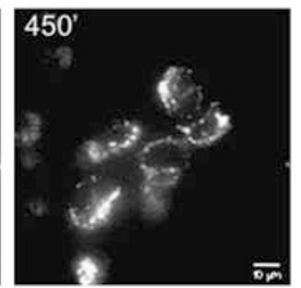

Cell death following TNFo treatment

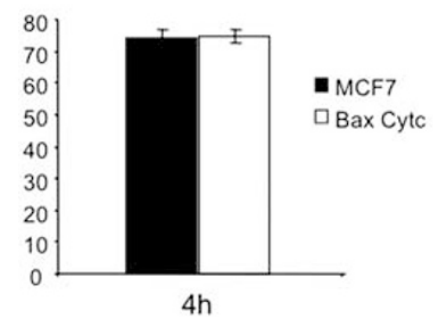

Figure 5 Stable expression of C-YFP-Bax and Cyt c-N-YFP. (a) MCF-7 cells were infected with lentiviruses containing C-YFP-HA-Bax and Cyt c-N-YFP. Stable clones were selected using blasticidine and the expression level was analyzed by Western blot using anti-Cyt $c$ and anti-HA antibodies. (b) MCF-7 cells stably expressing C-YFPHA-Bax and Cyt $c-N-Y F P$ were treated with etoposide $(100 \mu \mathrm{M})$ and followed using the DeltaVision system. (c) A total of $2 \times 10^{4}$ naive MCF-7 cells and cells that stably express C-YFP-HA-Bax and Cyt c-N-YFP were plated in black, 96-well plates and treated with staurosporine ( $1 \mu \mathrm{M}, 16$ and $22 \mathrm{~h})$, etoposide (100 $\mu \mathrm{M}, 16$ and $22 \mathrm{~h})$ and TNF $\alpha$ with $A c t D(4 \mathrm{~h})$. Cell viability was measured using the CellTiter Blue reagent and cell death was calculated relative to non-treated cells. No statistical significant differences were observed $(P>0.1)$

TNF $\alpha$ for the indicated time periods and measured cell death. No significant differences in the level of cell death could be detected between naive MCF7 cells and cells that stably express the Bax-Cyt $c$ split YFP pair (Figure 5c).

Hypoxia and chemotherapy-induced apoptosis in ovarian carcinoma cells. Human epithelial ovarian carcinoma cells (MLS) stably expressing the Bax-Cyt $c$ split YFP pair showed a significant elevation of YFP fluorescence in response to staurosporine and cisplatin treatment (Figure 6a). Hypoxiainduced apoptosis was evaluated in multicellular tumor spheroids derived from MLS human epithelial ovarian

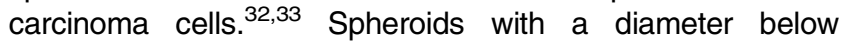
$100 \mu \mathrm{m}$ showed no YFP-positive cells (Figure 6b, upper panel), whereas larger spheroids $(>150 \mu \mathrm{m}$ in diameter) showed increased YFP fluorescence, mainly at the center of the spheroid (Figure 6b, middle and lower panels).

In vivo monitoring of chemotherapy-induced apoptosis was evaluated in a mixture of the stably expressing Bax-Cyt $c$ MLS cells $\left(2.7 \times 10^{6}\right.$ cells) and cells expressing the DsRed fluorescent protein $\left(1.3 \times 10^{6}\right.$ cells $)$ implanted into a dorsal skinfold window chamber in 8-week-old female nude mice. ${ }^{34}$ (Figure 6c) Ten days following tumor inoculation, apoptosis was induced by a single i.p. injection of $12 \mathrm{mg} / \mathrm{kg}$ cisplatin $(\mathrm{CP}){ }^{35}$ Intra-vital imaging of the tumors using a confocal microscope showed a significant increase in YFP-positive cells 22 and $32 \mathrm{~h}$ after $\mathrm{CP}$ injection, relative to that in saline-injected animals (Figure $6 \mathrm{c}$, middle and bottom panels). Immunohistochemistry staining using an anti-cleaved/active caspase-3 antibody showed a correlation between YFP-positive and caspase-3-positive cells (Figure 6c, bottom right panel).

\section{Discussion}

The ability to monitor apoptosis is important for the study of cancer progression and response to therapy. In this study we developed two novel tools, Bax-Bax and Bax-Cyt $c$ split YFP, which detect early events in mitochondrial apoptosis, both in vitro and in vivo.

The first tool detects Bax activation/homodimerization at the mitochondria. In healthy cells Bax resides in the cytosol in a closed/inactive conformation. In response to an apoptotic signal, Bax moves to the mitochondria and inserts into the OMM, resulting in a conformational change leading to its homodimerization/activation. Accordingly, the Bax-Bax split YFP pair residing in the cytosol of healthy MCF-7 cells generated a low YFP signal. Following an apoptotic stimulus, the YFP fluorescence was significantly increased and appeared in the mitochondria. Thus, the Bax-Bax split YFP pair faithfully recapitulates the known paradigm of Bax activation. Moreover, this YFP pair also faithfully reports the behavior of inactive/hyperactive mutants of Bax.

The second tool, the Bax-Cyt $c$ split YFP pair, detects the translocation of Bax to mitochondria and the release of Cyt $c$. Translocation of Bax to the mitochondria and its activation/ homodimerization in the OMM results in the release of Cyt $c$ from the inter-membrane space into the cytosol. Using the Bax-Cyt $c$ split YFP pair we detected a low YFP signal in healthy MCF-7 cells, and a significant increase in the signal when the cells were exposed to TNF $\alpha$ or menadione. This increase in the YFP signal was surprising as there are no reports in the literature describing an interaction between Bax and Cyt $c$. We assume that the increase in the YFP signal arises from the close proximity when residing in the same 
a

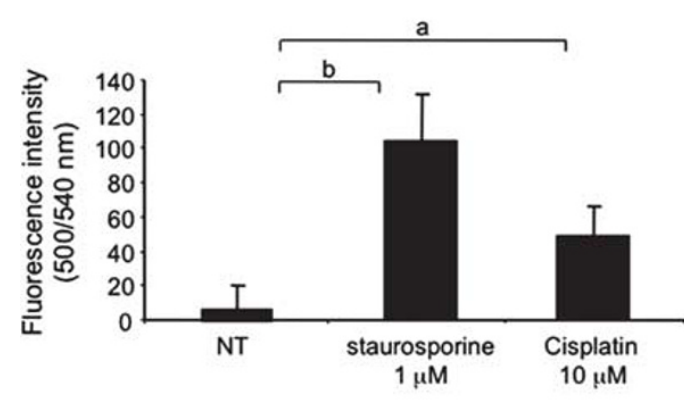

b

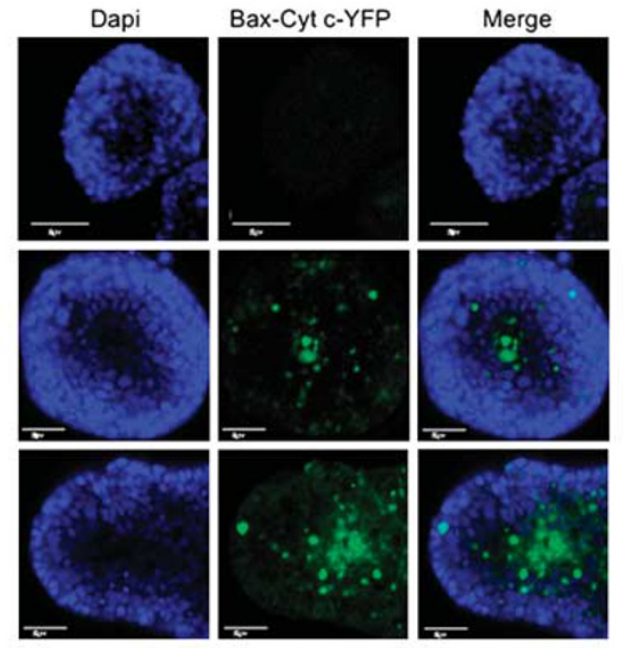

MLS spheroids expressing Bax-Cyt c-split YFP

C
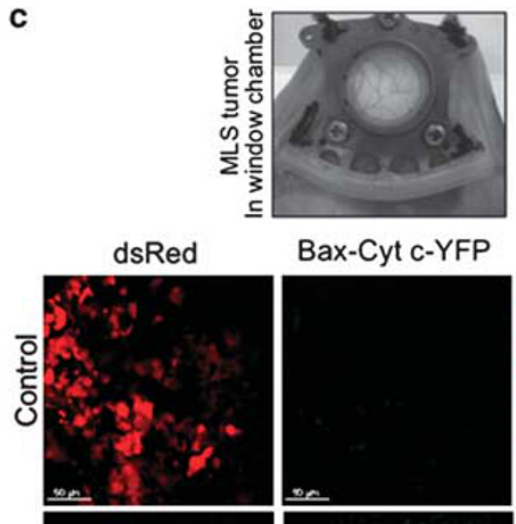

Bax-Cyt c-YFP
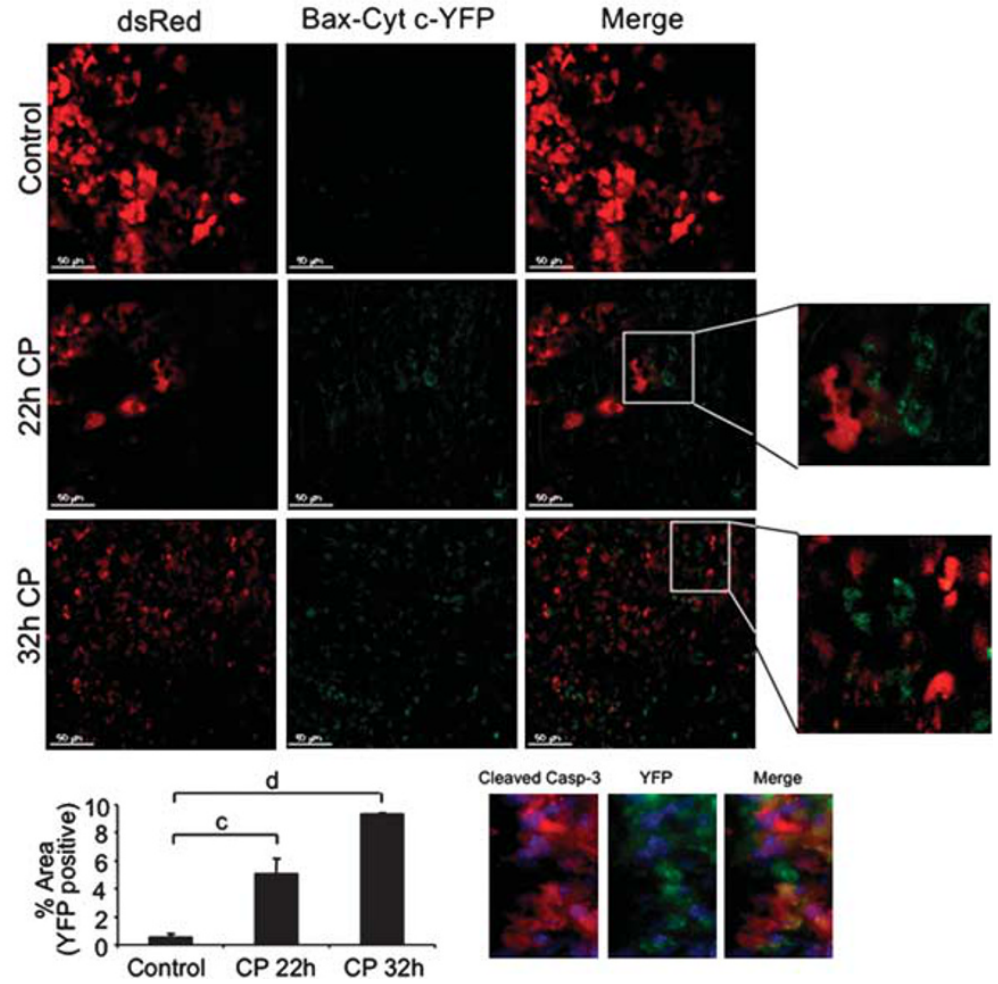

Figure 6 Bax-Cyt $c$ YFP complementation as a tool to detect tumor cell apoptosis. (a) MLS cells stably expressing Cyt c-N-YFP and C-YFP-Bax were seeded in a black, 96-well plate. Twenty-four hours later the cells were either left untreated (NT), or treated with $1 \mu \mathrm{M}$ staurosporine or $10 \mu \mathrm{M}$ cisplatin. YFP fluorescence was measured using a Synergy2 plate reader, using a YFP-specific filter set. ${ }^{a} P<0.05$; ${ }^{b} P<0.0001$. (b) MLS cells stably expressing Cyt $c-N-Y F P$ and C-YFP-HA-Bax were grown in a spinner flask for 10 days, fixed in $2.5 \%$ PFA and immersed in DAPI solution. The spheroids were imaged by confocal microscopy. (c) A mixture of MLS cells stably expressing either Cyt cN-YFP and C-YFP-HA-Bax $\left(2.7 \times 10^{6}\right)$, or DsRed $\left(1.3 \times 10^{6}\right)$, was injected intra-dermally into the center of a dorsal skinfold window chamber created in 8-week-old female CD-1 nude mice. Ten days later cisplatin was injected $(12 \mathrm{mg} / \mathrm{kg})$ and live-cell imaging was performed using a confocal microscope. The percentage of YFP-positive area was measured using the ImageJ software (bottom graph). ${ }^{c} P<0.002$; ${ }^{d} P<0.0001$. Tumors were removed and histological floating sections were stained using an anti-cleaved/ active caspase-3 antibody (red), and correlated with the YFP signal (green)

compartment (most likely the OMM) resulting in spontaneous complementation of the YFP fragments. Importantly, the increase in the Bax-Cyt $c$ YFP signal was closely followed by an increase in caspase activity, strongly suggesting that the increase in the Bax-Cyt $c$ YFP signal correlates with the induction of apoptosis. It is not clear whether Bax is involved in the cell death process induced by menadione, ${ }^{36,37}$ however the release of Cyt $c$ was shown in several studies. In the present study, YFP fluorescence upon menadione treatment was mostly distributed in the cytoplasm of the cells, suggesting that Cyt $c$ is released from the mitochondria and comes in close proximity with Bax in the cytosol. Of note, menadione is a known inducer of mitochondrial permeability transition (mPT), and thus our results also suggest that $\mathrm{mPT}$ is involved in the process of Bax-induced Cyt $c$ release.

The BH3-only Bid protein is a well-established activator of Bax. ${ }^{38}$ In response to an apoptotic stimulus, Bid is cleaved to $\mathrm{tBid}^{39}$ and tBid interacts with Bax resulting in the conformational changes described above. Unlike the Bax-Bax pair, in the Bax-Bid pair the background fluorescence in nonstimulated cells was very high, possibly owing to the toxic effect of Bid overexpression.

The Bax-Bax and Bax-Cyt c split YFP pairs could be used to show apoptosis in vivo. YFP fluorescence could be detected in damaged hepatocytes, and the number of YFPpositive cells increased after injection of anti-Fas antibody. Histological staining of active caspase-3 correlated with YFP fluorescence. The Bax-Cyt $c$ split YFP pair also proved to be powerful in the detection of hypoxia- and cisplatin-induced apoptosis of human ovarian carcinoma cells, in a threedimensional spheroid culture and in a skin flap tumor model.

In summary, we developed novel tools to image apoptosis in living cells and animals, taking advantage of the unique behavior of apoptotic proteins such as their conformational changes, oligomerizations and translocations. Cells can undergo apoptosis through mitochondria-dependent and -independent pathways. Adding the early markers of mitochondria-dependent commitment to apoptosis reported here, 
to the existing portfolio of apoptosis markers, will provide a non-invasive means for high-resolution, real-time monitoring of the apoptotic pathway. The applications of this method are variable, including high-throughput screening of novel regulators of apoptosis and new proapoptotic drugs, as well as monitoring of apoptosis in live animals by fluorescence intra-vital microscopy.

\begin{abstract}
Materials and Methods
Construction of plasmid expression vectors. The $\mathrm{N}$ - and C-terminal halves of the YFP expression vectors were a gift from Professor Michael Frohman (Stony Brook University, NY, USA). ${ }^{22}$ The N-YFP (amino acids 1-173) and C-YFP (amino acids 155-238) were cloned upstream to numerous genes of interest. Specifically, rat Cyt $c$ was cloned upstream to N-YFP in a pEFGP expression vector. $\mathrm{C}$-YFP or N-YFP was cloned upstream to HA mouse-Bax in the pcDNA-3 expression vector. C-YFP and N-YFP-HA from the N-YFP-HA-Bax mentioned above were also cloned into an empty pEGFP expression vector. For IF staining and analysis of sub-cellular localization of C-YFP-Bax and Cyt c-N-YFP, the two genes were also cloned in-frame to a Flag tag or an HA tag in the pcDNA-3 or pEGFP expression vectors, respectively. The GFP-caspase sensor was a gift from Professor Claudio Brancolini (Universita' di Udine, Italy). The GFP was replaced by mCherry by PCR using specific primers. The mCherry was a gift from Professor Roger Tsien (University of California, San Diego, CA, USA). The Smac-YFP vector was a gift from Professor Andrew Gilmore (University of Manchester). All the plasmids that were generated were verified by sequencing and Western blot analyses.
\end{abstract}

Cell culture, transfection and induction of apoptosis. MLS human epithelial ovarian carcinoma cells, generously provided by Professor RM Sutherland ${ }^{40}$ were cultured in $\alpha$-Eagle's minimum essential medium $(\alpha M E M)$, and MCF-7 human breast cancer cells (ATCC) were grown in DMEM. Media were supplemented with $10 \%$ fetal calf serum (FCS), $2 \mathrm{mM}$ L-glutamine, $1 \%$ penicillin/ streptomycin (Biological Industries, Beit Haemek, Israel). The MCF-7 cells were transfected using Lipofectamin-2000 (Invitrogen, Paisley, UK) in antibiotic-free medium according to the manufacturer's instructions. Twenty-four hours after transfection, the cells were treated with etoposide $(100 \mu \mathrm{M}$; Sigma, St Louis, MO, USA), staurosporine ( $1 \mu \mathrm{M}$; Sigma), menadione ( $30 \mu \mathrm{M}$; Sigma), cisplatin (10 $\mu \mathrm{M}$; Sigma), human TNF $\alpha$ (10 ng/ml; PeproTech, Rocky Hill, NJ, USA) and actinomycin D ( $2 \mu \mathrm{g} / \mathrm{ml}$; Sigma).

IF and imaging. Cells were plated in 24-well plates containing coverslips and transfected as described above. Twenty-four hour after transfection the cells were fixed with $3 \%$ paraformaldehyde in PBS and permeabilized with $0.2 \%$ Triton X-100/ PBS. The cells were immunostained with anti-HA (3F10; Roche, Mannheim, Germany) or anti-Flag (M2; Sigma) antibodies, followed by Cy3-conjugated goat anti-IgG antibodies (Jackson ImmunoResearch Laboratories, West Grove, PA, USA). Nuclei were stained with 4',6-diamidino-2-phenylindole dihydrochloride (DAPI, $10 \mu \mathrm{g} / \mathrm{ml}$; Sigma). Images were collected with an Olympus IX70 microscope, equipped with a DeltaVision imaging system, using a $\times 40$ PLAN-APO 1.42NA objective. Images were processed by constrained iterative deconvolution using the SoftWoRx software (Applied Precision, Issaquah, WA, USA).

Real-time imaging of apoptosis. MCF-7 cells were plated on 35-mm plates (ibidi GmbH, Germany) and transfected with the different DNA constructs. Twenty-four hours after transfection the cells were treated with a death signal (TNF $\alpha$ or etoposide) and visualized with an Olympus IX70 microscope, equipped with an incubator $\left(37^{\circ} \mathrm{C} 5 \% \mathrm{CO}_{2}\right)$ and a DeltaVision imaging system (Applied Precision), using a $\times 60$ objective. Images were collected every $20-45 \mathrm{~min}$ for $3-7 \mathrm{~h}$ and analyzed using the SoftWoRx software. Changes in YFP signal at a single-cell level were analyzed by converting a deconvolved time-lapse movie into a projection image. The maximal intensity of each cell was derived from a manually selected region of interest. Changes in fluorescence were also detected in MCF-7 cells cultured on a black, 96-well plate using a Synergy2 plate reader (Biotek, Winooski, VT, USA; excitation $500 \mathrm{~nm}$, emission $540 \mathrm{~nm}$ ). Twenty-four hours after transfection apoptosis was induced using etoposide, staurosporine or cisplatin in phenol red-free medium.
Caspase-3/7 activity assay. MCF-7 cells were cultured in a black, 96-well plate and transfected with C-YFP-HA-Bax and Cyt $c-N-Y F P$. Twenty-four hours after transfection the cells were either left untreated or treated with $50 \mu \mathrm{M}$ zVAD-fmk (Promega, Madison, WI, USA) to inhibit caspase activity (to reduce background activity), or treated with TNF $\alpha$ for various time periods. Caspase-3 activity was determined using the caspase-Glo 3/7 assay kit (Promega). A 100- $\mu$ l volume of the reagent was added directly to the culture medium, shaken briefly and incubated at room temperature for $1 \mathrm{~h}$. Luminescence was measured using the Wallac Victor2 1420 Multilabel plate reader (Perkin Elmer Life Sciences, Boston, MA, USA). Specific activity was calculated for each sample by subtracting the value obtained for the sample containing zVAD-fmk from the mean of the duplicate sample.

Generating MCF-7 and MLS stable lines. To stably express Cyt $c-$ N-YFP and C-YFP-Bax in MCF-7 and MLS cells we used the pLenti6/V5 GateWay system from Invitrogen (Paisley, UK). Briefly, the C-YFP-Bax and Cyt $c-N-Y F P$ were independently cloned into the pCR8/GW/TOPOTA cloning system (K2500-20) and then introduced into the pLenti6/V5 vector using a GateWay LR-clonase (11791-020). Lentiviruses were generated in HEK293 FT cells using the ViraPower lentiviral expression system (K4975-00), according to the manufacturer's instructions. Stable clones were selected using blasticidin $\left(5 \mu \mathrm{g} \mathrm{ml}^{-1}\right.$; Fluka, Sigma) and expression was analyzed by Western blot analysis. Cell viability was measured in 96-well plates using CellTiter Blue reagent from Promega.

Hydrodynamic injections for hepatocellular somatic gene transfer. All experiments on animals were approved by the Weizmann Institutional Animal Care and Use Committee (IACUC). For in vivo mouse experiments a total of $30 \mu \mathrm{g}$ of mCherry caspase sensor and the split YFP fusion DNA were mixed with solution-A $\left(2.7 \mathrm{mM} \mathrm{KCl}, 1.47 \mathrm{mM} \mathrm{KH}_{2} \mathrm{PO}_{4}, 139 \mathrm{mM} \mathrm{NaCl}\right.$, $\left.8.1 \mathrm{mM} \mathrm{Na}_{2} \mathrm{HPO}_{4}(\mathrm{pH} 7.4)\right)$ to a total volume of up to $10 \%$ of the body weight of mouse. The entire DNA solution was injected rapidly into the tail vein of awake FVB male mice (age 8-9 weeks, $n=4$ ) in a restraining device, as reported previously. ${ }^{30}$ To induce liver apoptosis, a single i.p. dose of $0.55 \mu \mathrm{g} / \mathrm{g}$ anti-Fas antibody (BD Pharmingen, San Diego, CA, USA) was injected.

For visualization of YFP fluorescence, the livers were excised. Half of the liver was imaged immediately by two-photon microscopy (2PM; Zeiss LSM 510 META NLO; equipped with a broadband Mai Tai-HP femtosecond single-box tunable Ti-sapphire oscillator, with automated broadband wavelength tuning 700-1020 nm from Spectraphysics, for two-photon excitation).

For histological analysis, liver samples were fixed in $2.5 \%$ paraformaldehyde (PFA), kept at room temperature for $24 \mathrm{~h}$, followed by incubation in $30 \%$ sucrose for at least $48 \mathrm{~h}$ at $4{ }^{\circ} \mathrm{C}$. Sequential $20-\mu \mathrm{m}$ microtome sections were collected and stored at $4^{\circ} \mathrm{C}$ in PBS.

Spheroid culture. MLS human epithelial ovarian carcinoma cells were cultured in $\alpha \mathrm{MEM}$ supplemented with $10 \%$ FCS and antibiotics. ${ }^{32}$ Aggregation of cells into spheroids was initiated by plating the cells from confluent cultures into agar-coated plates. After $48 \mathrm{~h}$, the spheroids were transferred to a $250-\mathrm{ml}$ spinner flask (Bellco, Vineland, NJ, USA) at a spinning rate of 80 r.p.m., where every $72 \mathrm{~h}$ for 10 days the medium was changed and a mixture of $95 \%$ air and $5 \%$ carbon dioxide was blown over the medium for $5 \mathrm{~min}$.

In vivo imaging of tumors in dorsal skinfold window chamber model. Window chambers were implanted into a dorsal skinfold of 8-week-old CD-1 nude female mice as was described previously. ${ }^{34}$ Tumors were initiated $24 \mathrm{~h}$ after surgery by co-injection of $4 \times 10^{6}$ (in $30 \mu$ l of PBS) of MLS cells stably expressing either DsRed (one-third) or the Bax-Cyt c split YFP pair (two-third) to the center of the chamber. The tumors were imaged starting at 10 days after injection, under isoflurane anesthesia, by confocal microscopy (Zeiss LSM 510 META NLO; $\times 20$ lens). Apoptosis was induced by i.p. injection of cisplatin $\left(12 \mathrm{mg} \mathrm{kg}^{-1,35}\right.$ dissolved in $0.9 \% \mathrm{NaCl})$. The percentage of YFP-coverage area was calculated using ImageJ (National Institutes of Health; http://rsb.info.nih.gov/ij/).

Histological analysis and immunohistochemical staining. For immunohistochemical staining we used rabbit anti-active caspase-3 antibodies (1:50; Cell Signaling, Danvers, MA, USA), anti-rabbit biotin antibodies $(1: 200)$ and streptavidin-CY5 (1:100) (Jackson ImmunoResearch Laboratories). For DNA counterstaining, we used Hoechst $(1 \mu \mathrm{g} / \mathrm{ml}$; Molecular Probes, Invitrogen, Paisley, UK). Unstained floating sections were visualized using a Zeiss AXIO observer fluorescent microscope using a $\times 20$ objective. Images were obtained with an 
Olympus DP72 camera and analyzed using the Olympus cell ${ }^{A}$ software. Immunohistochemically stained sections were collected using an Olympus IX70 microscope, equipped with a DeltaVision imaging system, using a $\times 40$ PLAN-APO 1.42NA objective.

Statistical analysis. Data are presented as the average \pm S.D. of multiple $(n \geq 3)$ transfections. Student's unpaired two-tailed $t$-test was performed using the statistical analysis functions of Microsoft Excel. Differences were considered statistically significant at $P<0.05$.

\section{Conflict of Interest}

The authors declare no conflict of interest.

Acknowledgements. Michal Neeman is incumbent of the Helen and Morris Mauerberger Chair, and Atan Gross is incumbent of the Armour Family Career Development Chair of Cancer Research. This work was supported by the USA NIH R01 CA75334, The European Commission FP7 Integrated Project ENCITE and the 7th Framework European Research Council Advanced grant 232640-IMAGO (to $\mathrm{MN})$, and by the USA-Israel Binational Science Foundation and the Israel Science Foundation (to $A G$ ).

1. Meier P. Finch A, Evan G. Apoptosis in development. Nature 2000; 407: 796-801.

2. Banihashemi B, Vlad R, Debeljevic B, Giles A, Kolios MC, Czarnota GJ. Ultrasound imaging of apoptosis in tumor response: novel preclinical monitoring of photodynamic therapy effects. Cancer Res 2008; 68: 8590-8596.

3. Moffat BA, Chenevert TL, Lawrence TS, Meyer CR, Johnson TD, Dong Q et al. Functional diffusion map: a noninvasive MRI biomarker for early stratification of clinical brain tumor response. Proc Natl Acad Sci USA 2005; 102: 5524-5529.

4. Bullok KE, Maxwell D, Kesarwala AH, Gammon S, Prior JL, Snow M et al. Biochemical and in vivo characterization of a small, membrane-permeant, caspase-activatable far-red fluorescent peptide for imaging apoptosis. Biochemistry 2007; 46: 4055-4065.

5. Laxman B, Hall DE, Bhojani MS, Hamstra DA, Chenevert TL, Ross BD et al. Noninvasive real-time imaging of apoptosis. Proc Natl Acad Sci USA 2002; 99: 16551-16555.

6. Bauer C, Bauder-Wuest U, Mier W, Haberkorn U, Eisenhut M. 1311-labeled peptides as caspase substrates for apoptosis imaging. J Nucl Med 2005; 46: 1066-1074.

7. Thornberry NA, Rano TA, Peterson EP, Rasper DM, Timkey T, Garcia-Calvo M et al. A combinatorial approach defines specificities of members of the caspase family and granzyme B. Functional relationships established for key mediators of apoptosis. J Biol Chem 1997; 272: 17907-17911

8. Zhang G, Gurtu V, Kain SR, Yan G. Early detection of apoptosis using a fluorescent conjugate of annexin V. Biotechniques 1997; 23: 525-531.

9. Zhao M, Beauregard DA, Loizou L, Davletov B, Brindle KM. Non-invasive detection of apoptosis using magnetic resonance imaging and a targeted contrast agent. Nat Med 2001; 7: 1241-1244.

10. Chowdhury I, Tharakan B, Bhat GK. Caspases - an update. Comp Biochem Physiol B Biochem Mol Biol 2008; 151: 10-27.

11. Smrz D, Draberova L, Draber P. Non-apoptotic phosphatidylserine externalization induced by engagement of glycosylphosphatidylinositol-anchored proteins. J Biol Chem 2007; 282 10487-10497.

12. Youle RJ, Strasser A. The BCL-2 protein family: opposing activities that mediate cell death Nat Rev Mol Cell Biol 2008; 9: 47-59.

13. Hsu YT, Youle RJ. Nonionic detergents induce dimerization among members of the $\mathrm{Bcl}-2$ family. J Biol Chem 1997; 272: 13829-13834.

14. Suzuki M, Youle RJ, Tjandra N. Structure of Bax: coregulation of dimer formation and intracellular localization. Cell 2000; 103: 645-654.

15. Goping IS, Gross A, Lavoie JN, Nguyen M, Jemmerson R, Roth $\mathrm{K}$ et al. Regulated targeting of BAX to mitochondria. J Cell Biol 1998; 143: 207-215.

16. Hsu YT, Wolter KG, Youle RJ. Cytosol-to-membrane redistribution of Bax and Bcl-X(L) during apoptosis. Proc Natl Acad Sci USA 1997; 94: 3668-3672.

17. Desagher S, Osen-Sand A, Nichols A, Eskes R, Montessuit S, Lauper S et al. Bid-induced conformational change of Bax is responsible for mitochondrial cytochrome $c$ release during apoptosis. J Cell Biol 1999; 144: 891-901.

18. Gross A, Jockel J, Wei MC, Korsmeyer SJ. Enforced dimerization of BAX results in its translocation, mitochondrial dysfunction and apoptosis. EMBO J 1998; 17: 3878-3885.

19. Lovell JF, Billen LP, Bindner S, Shamas-Din A, Fradin C, Leber B et al. Membrane binding by tBid initiates an ordered series of events culminating in membrane permeabilization by Bax. Cell 2008; 135: 1074-1084.

20. Acehan D, Jiang X, Morgan DG, Heuser JE, Wang X, Akey CW. Three-dimensional structure of the apoptosome: implications for assembly, procaspase-9 binding, and activation. Mol Cell 2002; 9: 423-432.

21. Mahajan NP, Linder K, Berry G, Gordon GW, Heim R, Herman B. Bcl-2 and Bax interactions in mitochondria probed with green fluorescent protein and fluorescence resonance energy transfer. Nat Biotechnol 1998; 16: 547-552.

22. Choi SY, Huang P, Jenkins GM, Chan DC, Schiller J, Frohman MA. A common lipid links Mfn-mediated mitochondrial fusion and SNARE-regulated exocytosis. Nat Cell Biol 2006; 8: $1255-1262$

23. Hu CD, Chinenov Y, Kerppola TK. Visualization of interactions among bZIP and Rel family proteins in living cells using bimolecular fluorescence complementation. Mol Cell 2002; 9: 789-798.

24. Upton JP, Valentijn AJ, Zhang L, Gilmore AP. The N-terminal conformation of Bax regulates cell commitment to apoptosis. Cell Death Differ 2007; 14: 932-942.

25. Schinzel A, Kaufmann T, Schuler M, Martinalbo J, Grubb D, Borner C. Conformational control of Bax localization and apoptotic activity by Pro168. J Cell Biol 2004; 164: 1021-1032.

26. Nechushtan A, Smith CL, Hsu YT, Youle RJ. Conformation of the Bax C-terminus regulates subcellular location and cell death. EMBO J 1999; 18: 2330-2341.

27. Priault M, Cartron PF, Camougrand N, Antonsson B, Vallette FM, Manon S. Investigation of the role of the C-terminus of Bax and of tc-Bid on Bax interaction with yeast mitochondria. Cell Death Differ 2003; 10: 1068-1077.

28. Henderson CJ, Aleo E, Fontanini A, Maestro R, Paroni G, Brancolini C. Caspase activation and apoptosis in response to proteasome inhibitors. Cell Death Differ 2005; 12: 1240-1254.

29. Shu X, Shaner NC, Yarbrough CA, Tsien RY, Remington SJ. Novel chromophores and buried charges control color in mFruits. Biochemistry 2006; 45: 9639-9647.

30. Villalobos V, Naik S, Piwnica-Worms D. Detection of protein-protein interactions in live cells and animals with split firefly luciferase protein fragment complementation. Methods Mol Biol 2008; 439: 339-352.

31. Nishimura $\mathrm{Y}$, Hirabayashi $\mathrm{Y}$, Matsuzaki $\mathrm{Y}$, Musette $\mathrm{P}$, Ishii A, Nakauchi $\mathrm{H}$ et al. In vivo analysis of Fas antigen-mediated apoptosis: effects of agonistic anti-mouse Fas mAb on thymus, spleen and liver. Int Immunol 1997; 9: 307-316.

32. Schiffenbauer YS, Abramovitch R, Meir G, Nevo N, Holzinger M, Itin A et al. Loss of ovarian function promotes angiogenesis in human ovarian carcinoma. Proc Natl Acad Sci USA 1997; 94: 13203-13208.

33. Hauptmann S, Gebauer-Hartung P, Leclere A, Denkert C, Pest S, Klosterhalfen B et al. Induction of apoptosis in the centre of multicellular tumour spheroids of colorectal adenocarcinomas - involvement of CD95 pathway and differentiation. Apoptosis 1998; 3 : 267-279

34. Cohen B, Addadi Y, Sapoznik S, Meir G, Kalchenko V, Harmelin A et al. Transcriptional regulation of vascular endothelial growth factor $C$ by oxidative and thermal stress is mediated by lens epithelium-derived growth factor/p75. Neoplasia 2009; 11: 921-933.

35. Meyn RE, Stephens LC, Hunter NR, Milas L. Apoptosis in murine tumors treated with chemotherapy agents. Anticancer Drugs 1995; 6: 443-450.

36. Loor G, Kondapalli J, Schriewer JM, Chandel NS, Vanden Hoek TL, Schumacker PT. Menadione triggers cell death through ROS-dependent mechanisms involving PARP activation without requiring apoptosis. Free Radic Biol Med 2010; 49: 1925-1936.

37. Hou Q, Hsu YT. Bax translocates from cytosol to mitochondria in cardiac cells during apoptosis: development of a GFP-Bax-stable H9c2 cell line for apoptosis analysis. Am J Physiol Heart Circ Physiol 2005; 289: H477-H487.

38. Roucou X, Montessuit S, Antonsson B, Martinou JC. Bax oligomerization in mitochondrial membranes requires tBid (caspase-8-cleaved Bid) and a mitochondrial protein. Biochem $J$ 2002; 368 (Part 3): 915-921.

39. Gross A, Yin XM, Wang K, Wei MC, Jockel J, Milliman C et al. Caspase cleaved BID targets mitochondria and is required for cytochrome $c$ release, while $B C L-X L$ prevents this release but not tumor necrosis factor-R1/Fas death. J Biol Chem 1999; 274: 1156-1163.

40. Rofstad EK, Sutherland RM. Growth and radiation sensitivity of the MLS human ovarian carcinoma cell line grown as multicellular spheroids and xenografted tumours. Br J Cancer 1989; 59: 28-35

(c)

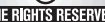

Cell Death and Disease is an open-access journal published by Nature Publishing Group. This work is licensed under the Creative Commons Attribution-Noncommercial-No Derivative Works 3.0 Unported License. To view a copy of this license, visit http://creativecommons.org/licenses/by-nc-nd/3.0/

\section{Supplementary Information accompanies the paper on Cell Death and Disease website (http://www.nature.com/cddis)}

